\title{
Clinical predictors of hemorrhagic transformation after thrombolytic therapy an Egyptian center experience
}

\author{
Hazem Kamal Ibrahim, Mohamed Khodery. \\ Department of Neurology and psychological medicine, Sohag university, Egypt
}

Background: thrombolysis is the only approved pharmacological treatment for acute stroke. It acts as a mediator of recanalization and sometimes causes secondary hemorrhage depending on the size of the ischemic lesion, little is known about the efficacy and hemorrhagic complication of thrombolytic therapy, we aim to identify clinical predictors of hemorrhagic transformation.

Methods: a cross-sectional comparative study in Sohag University Hospital for 1226 acute ischemic stroke patient admitted to our department from $1^{\text {st }}$ April 2019 till $31^{\text {st }}$ March 2020, of the 21 patients treated with thrombolytic therapy further subdivided into two groups; (Group A): patients who didn't complicate with hemorrhagic transformation and (group B) patients who developed hemorrhagic transformation after receiving intravenous thrombolysis, the correlation between hemorrhagic transformation and clinical characteristic, vascular risk factors, and demographic data is done.

Results: We found a small percentage (1.7\%) of acute ischemic stroke patients receiving thrombolytic therapy. Old age, ischemic heart disease, high admission NIHSS on admission, and NIHSS $24 \mathrm{~h}$ after admission are associated with hemorrhagic transformation with p-value (0.024), (0.048), (0.085), and (0.001) respectively. Patients treated with thrombolytic therapy have statistically significant improvement in NIHSS $24 \mathrm{~h}$ after admission in comparison to the admission NIHSS with a p-value $(<0.001)$.

Conclusion: Elderly, high NIHSS on admission, and tachycardia are associated with hemorrhagic transformation following treatment with IV thrombolytic therapy, Despite the updated management of acute ischemic stroke only a small percentage (1.7\%) of acute ischemic stroke patients receiving thrombolytic therapy, this in need of further investigations.

Keywords: thrombolytic therapy, stroke, Sohag, hemorrhagic transformation

List of abbreviation NIHSS; National Institute Of Health Stroke Scale, TOAST; trials of Org 10172 in acute stroke treatment, r-tPA; tissue plasminogen activator, AHA; the American Heart Association, ASAT; American Stroke Association, HI; Hemorrhagic Infarctions, HT; Hemorrhagic transformation, ECASS; European Cooperative Acute Stroke Study, PH; parenchymal hemorrhage, SICH; symptomatic intracranial hemorrhage

\section{Introduction}

The Middle East North Africa is made up of 17 countries and stretches from East Asia to Morocco in the West. In 2017 the area has an estimated 411 million people. In the absence of highquality data from the area about stroke incidence and prevalence, it is difficult to establish the exact burden of this 
devastating disease. But because these countries are undergoing epidemiological research, the burden of noncommunicable diseases is increasing and an updated review of the literature available suggests an increase in the incidence of stroke and mortality in the region ${ }^{l}$.

Unfortunately, stroke care in this area is still in its infancy and while alteplase was approved more than two decades ago, this therapy is supported by very few centers. Until 2012, there were less than 10 centers in this area that treated patients with intravenous thrombolysis for acute ischemic stroke and utilization of reperfusion therapies for stroke $<1 \%$ in Egypt ${ }^{2}$ updating guidelines and recommendations for assessing the consistency of the Stroke Survival Chain, in which remains $<1$ percent. The American Heart Association / American Stroke Association (AHA / ASA) is constantly prehospital onset to arrival time (OAT) and intra-hospital to- needle time (DNT) do not exceed 35 and $60 \mathrm{~min}$, respectively $^{3}$. In developed countries, most hospitals are adjusting their programs to shorten the time for stroke patients to fit these guidelines in administrative procedures, clinical assessment, imagery, and laboratory investigation ${ }^{4}$. Thus, stroke treatment protocols in developing countries face many difficulties among their stroke patients, including low stroke understanding, poor finances, and complicated administrative procedures with resulting in higher mortalities and greater disabilities ${ }^{5}$.

Hemorrhagic transformations (HTs) were classified according to clinical and radiological criteria. HI1 was defined as small petechiae along the margins of the infarct; HI2, as confluent petechiae within the infarcted area, but no spaceoccupying effect; $\mathrm{PH} 1$, as blood clots in $\leq 30 \%$ of the infarcted area with some slight space-occupying effect; and $\mathrm{PH} 2$, as blood clots in .>30\% of the infarcted area with a substantial space-occupying effect ${ }^{7}$.

\section{Patients and methods}

Study design and patients

We conducted a cross-sectional retrospective cohort study enrolling patient hospitalized for acute ischemic stroke at the Department of Neurology, Sohag University Hospital; data were collected between $1^{\text {st }}$ April 2019 and $31^{\text {st }}$ March 2020. One thousand two hundred twenty-six patients with acute ischemic stroke have been admitted during the period of the study however one thousand one hundred ninety-seven twenty-nine patients were excluded as they presented to the hospital after the time window for thrombolysis and eight patients had been excluded due to missed data, transfer or had contradictions for thrombolysis. So only twent$\mathrm{y}$-one patients received thrombolytic therapy, furtherly subdivided into two groups according to treatment response: nineteen patients didn't develop hemorrhagic transformation (group A) and two patients developed hemorrhagic transformation (group B) flow chart is shown in Figure1

\section{Ethical consideration}

The study was approved by the local Ethics committee of the Sohag Faculty of Medicine. all patients signed written consent.

\section{Variable definitions}

Strokes were tested and verified if neurological defects lasted longer than 24 hours and/or focal ischemic damage suggested by cranial computed tomography or magnetic resonance imaging was detected ${ }^{6}$ Intracranial hemorrhages was identified as symptomatic (SICH) if the patient experienced clinical deterioration causing a 4-point increase in the National Institutes for Health Stroke Scale (NIHSS) score and the hemorrhage was likely to cause clinical deterioration ${ }^{7}$ 
For the whole participants, we analyzed medical and socio-economic variables, including age, sex, cerebrovascular risk factors, current smoking, stroke intensity (as assessed by the National Institutes of Health Stroke Scale ) on admission and 24 after admission, and the subtype of stroke according to the TOAST criteria ${ }^{8}$

\section{Data analysis}

Descriptive statistics of patients presented using frequencies and percentages for categorical variables, and the median range for continuous variables, using the $\chi 2$ test or Fisher's exact test for categorical variables. Mann-Whitney U test was used for continuous variables.

Both analyses were conducted using SPSS for version 19.0 of Windows (SPSS Inc., Chicago, Illinois) and Graph Pad Prism 8.0 (Graph Pad Software Inc., La Jolla, California, USA). A P value $<0.05$ has been regarded as statistically significant.

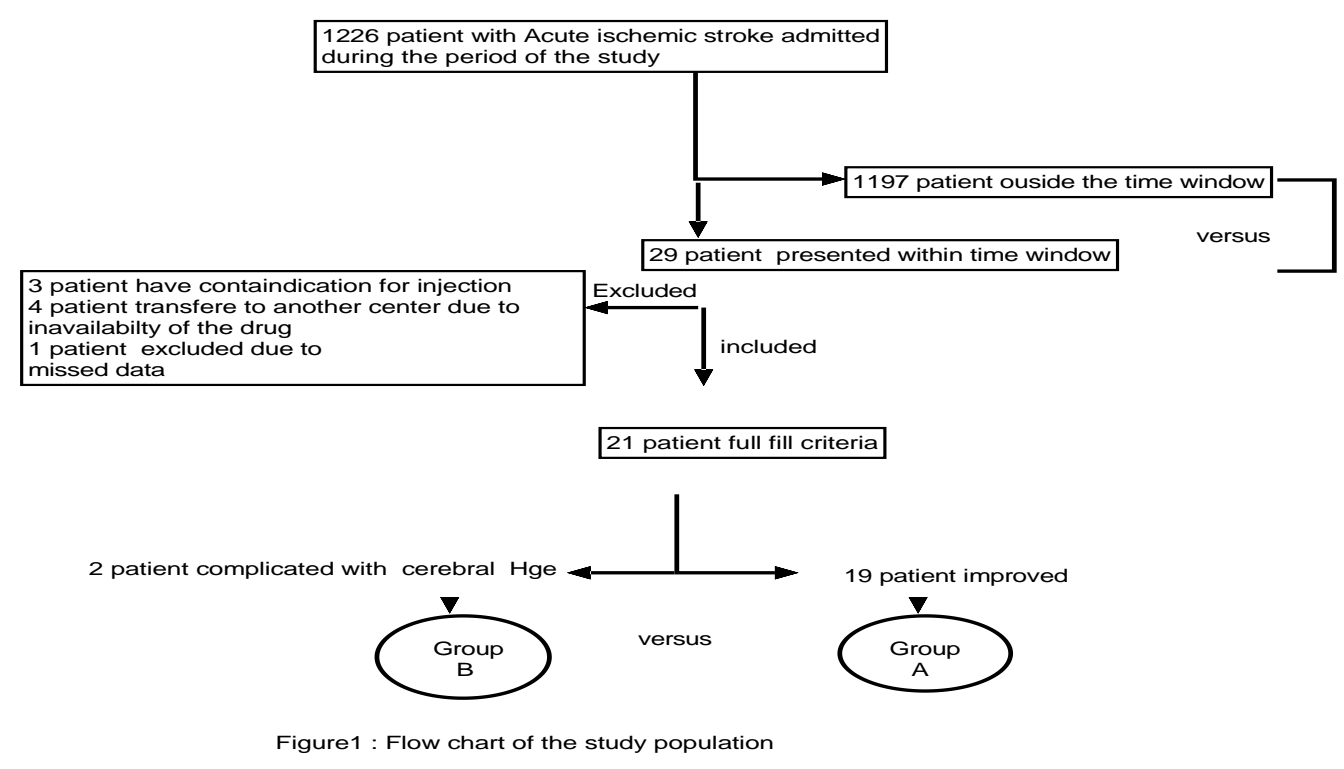

\section{Results of the study}

The mean age of our study population was around $59 \pm 12$.1years, Males are slightly more than females ( $62 \%$ versus $38 \%$ ). Most cases were from rural regions $(77 \%)$, and all of them were married (100\%). All cases were right-handed $(100 \%)$. Hypertension was found in more than one-third of our cases $(38 \%)$. Diabetes mellitus was found in $9.5 \%$ of our cases and more than half of the patients were nonsmokers ( $81 \%$ ). Regarding cardiac diseases, RHD is the most common heart disease found $(38 \%)$ followed by IHD (24\%), then AF (4.8\%), and lastly CHF only $4.8 \%$., As regard, clinical stroke scales the mean admission NIHSS was $11 \pm 5$, and the median NIHSS $24 \mathrm{~h}$ after admission was 6 and the range was 22 .

Regarding etiology of ischemic stroke small vessel disease "S" was the most common TOAST classification, the subtype is seen in $28.6 \%$ of cases, and cardioembolic " $\mathrm{C}$ " in $28.6 \%$ then large artery atherosclerosis "L" $23.8 \%$ and stroke of undetermined etiology " $U$ " in $19 \%$, Cerebral hemorrhage followed treatment was found in $9.5 \%$ of our population (Table 1) 
SOHAG MEDICAL JOURNAL

Vol. 24 No. 3 July 2020
Clinical predictors of hemorrhagic transformation Hazem Kamal Ibrahim

\begin{tabular}{|l|l|}
\hline Patient characteristic & Value \\
\hline Age, Mean \pm SD(years) & $59 \pm 12$ \\
\hline Sex female, n (\%) & $13(62 \%)$ \\
\hline Residence urban n (\%) & $7(33 \%)$ \\
\hline Hypertensive, n (\%) & $8(38 \%)$ \\
\hline Diabetic, n (\%) & $2(9.5 \%)$ \\
\hline IHD, n (\%) & $5(23.8 \%)$ \\
\hline CHF, n (\%) & $1(4.8 \%)$ \\
AF, n (\%) & $1(4.8 \%)$ \\
RHD, $(\%)$ & $8(38 \%)$ \\
\hline Current smoker, n (\%) & $2(9.5 \%)$ \\
\hline X smoker & $2(9.5 \%)$ \\
\hline TOAST subtype & \\
small vessel, $\mathrm{n}(\%)$ & $6(28.6 \%)$ \\
\hline, cardioembolic, $\mathrm{n}(\%)$ & $6(28.6 \%)$ \\
\hline, large vessel, $\mathrm{n}(\%)$ & $5(23.8 \%)$ \\
\hline Undetermined etiology, $\mathrm{n}(\%)$ & $4(19 \%)$ \\
\hline Hemorrhagic transformation, yes, n (\%) & $2(9.5 \%)$ \\
No, n (\%) & $19(90.5 \%$ \\
\hline Anterior circulation, $\mathrm{n}(\%)$ & $18(85.7 \%)$ \\
\hline Admission NIHSS, Mean \pm SD & $11 \pm 5$ \\
\hline 24 h NIHSS, Median (range) & $6(22)$ \\
\hline Systolic BP, Mean \pm SD & $131 \pm 20$ \\
\hline Diastolic, BP, Mean \pm SD & $83 \pm 11$ \\
\hline HR, Mean \pm SD & $82 \pm 22$ \\
\hline
\end{tabular}

Table1: clinical characteristic of study populationns $(\mathrm{N}=21)$

NIHSS; National Institute Of Health Stroke Scale, TOAST; trials of Org 10172 in acute stroke treatment, IHD; ischemic heart disease, CHD; congestive heart failure, RHD; rheumatic heart disease, HR; heart rate, AF; atrial fibrillation

The age is significantly higher in group $\mathrm{B}$ compared to group A with a p-value (0.024), patient in group B has higher admission NIHSS compared to group Abut the p-value is not statistically significant (0.085). NIHSS 24 hours after admission was statistically significantly lower in group A in comparison to group B with a p-value (0.001).
Tachycardia on admission and the history of IHD is significantly higher in the group compared to group A with $\mathrm{p}$ value $(0.025)$, and $(0.048)$ respectively, $\mathrm{CHF}$ is relatively higher in group B compared to group A but the p-value not statistically significant (0.09) ( Table 2)

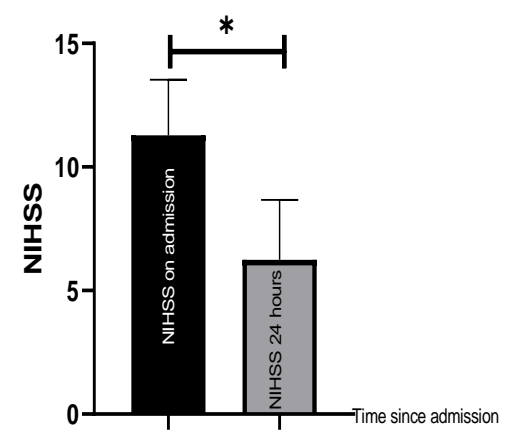

Figure 2: NIHSS on admission and 24 hours after

There was a statistically significant difference between admission and $24 \mathrm{~h}$ NIHSS $\mathrm{p}$ value was $(<0.001)$ figure 2 
SOHAG MEDICAL JOURNAL

Vol. 24 No. 3 July 2020

\begin{tabular}{|l|l|l|l|}
\hline & $\begin{array}{l}\text { Group A } \\
\mathrm{N}=19\end{array}$ & $\begin{array}{l}\text { Group B } \\
\mathrm{N}=2\end{array}$ & P-value \\
\hline Age & $57 \pm 11$ & $77 \pm 3$ & $\mathbf{0 . 0 2 4}$ \\
\hline admission NIHSS & $11 \pm 4$ & $17 \pm 7$ & 0.085 \\
\hline NIHSS 24h & $5 \pm 4$ & $17 \pm 7$ & $\mathbf{0 . 0 0 1}$ \\
\hline HR & $78 \pm 15$ & $112 \pm 32$ & $\mathbf{0 . 0 2 5}$ \\
\hline Systolic BP & $134 \pm 22$ & $125 \pm 21$ & 0.61 \\
\hline Diastolic BP & $82 \pm 11$ & $80 \pm 14$ & 0.76 \\
\hline Sex, Female & $11(52.4 \%)$ & $2(9.5 \%)$ & 0.50 \\
\hline Rural, residence & $13(61.9)$ & $1(4.8 \%)$ & 0.56 \\
\hline IHD, & $3(14.3 \%)$ & $2(9.5 \%)$ & $\mathbf{0 . 0 4 8}$ \\
\hline CHF & $0(0 \%)$ & $1(4.8 \%)$ & 0.095 \\
\hline DM & $1(48 \%)$ & $1(48 \%)$ & 0.18 \\
\hline Anterior circulation & $16(76 \%)$ & $2(9.5 \%)$ & 1 \\
\hline Hypertensive & $8(38 \%)$ & $0(0 \%)$ & 0.37 \\
\hline Small vessel & $5(23.8 \%)$ & $1(4.8 \%)$ & 0.50 \\
\hline Non-small vessel & $14(66.7 \%)$ & $1(4.8 \%)$ & 0.50 \\
\hline
\end{tabular}

Table 2 differences between the two groups

NIHSS; National Institute Of Health Stroke Scale, IHD; ischemic heart disease, CHD; congestive heart failure,, HR; heart rate, DM; diabetes millets

\section{Discussion}

To the best of our knowledge, this is the first study to identify clinical characterristics and complications of intravenous thrombolytic therapy in upper Egypt. We found that only $2.3 \%$ of the patients presented within the time window and only about $1.7 \%$ of acute ischemic stroke patients received thrombolytic therapy and this is in line with previous Egyptian publications. ${ }^{2}$ But less than international studies.

We have a relatively high percentage of hemorrhagic transformation (9.5\%).

Our result in line with Larrue et al 2001 who reported that; extreme HTs were typical in patients treated with $\mathrm{r}$ tPA. Based on the term used (SICH or $\mathrm{PH}$ ), between $8.8 \%$ and $11.8 \%$ of r-tPA patients had a serious hemorrhagic transformation.

In contrast, as previously observed in $\operatorname{ECASS}^{10}$, Hemorrhagic Infarctions were not more frequent in r-tPA-treated patients. Furthermore, among patients with HI, the odds for bad outcomes were significantly lower in those given r-tPA than in those receiving placebo, suggesting that r-tPA may benefit some patients despite minor degrees of HT.
Hemorrhagic transformation relatively higher in the elderly and this is in line with the previous publication ${ }^{7}$, but we found that the mean age of hemorrhagic transformation is relatively younger compared to previous studies.

High admission NIHSS was associated with hemorrhagic transformation, and this agreed with previous publications. ${ }^{9}$ History of IHD is associated with hemorrhagic transformation.

There was a statistically significant difference between admission and $24 \mathrm{~h}$ NIHSS reflecting the efficacy of thrombolytic therapy in our study population and this is worldwide, and FDA approved

\section{The limitation of the study}

A relatively small sample size makes it difficult to generalize our results.

\section{Strength of the study}

The first author is a qualified stroke consultant, and the last author is a qualified stroke physician (ESO member and NIHSS certified) and they were in contact with all patients on the study. 


\section{Conclusion}

Hemorrhagic transformation is a serious complication of thrombolytic therapy. elderly, high NIHSS on admission, ischemic heart disease, and tachycardia is associated with hemorrhagic transformation after r-tPA treatment, but these risk factors need to be validated on an independent data set. Only a small percentage $(1.7 \%)$ of acute ischemic stroke patients receiving thrombolytic therapy, this in need of further investigations.

\section{Source of funding}

Non

\section{Acknowledgment; Non}

Conflict of interest; the authors report no conflict of interest

None.

\section{References}

1 V.L. Feigin, B. Norrving, G.A. Mensah, Global burden of stroke, Circ. Res. 120 (3) (2017) 439-448.

2 Magd Fouad Zakaria, Hany Aref, Azza Abd ElNasser, Nagia Fahmy, et al, Egyptian experience in increasing utilizeation of reperfusion therapies in acute ischemic stroke, International Journal of Stroke, 2017:4, 1-5

3 G.C. Fonarow, E.E. Smith, J.L. Saver, et al., Timeliness of tissue-type plasminogen activator therapy in acute ischemic stroke patient characteristics, hospital factors, and outcomes associated with door-to-needle times within 60 minutes, Circulation 123 (2011) 750-758, https://doi.org/.1161/CIRCULATIONA HA. 110.974675 .

4 L.R. Caplan, Primary stroke centers vs comprehensive stroke centers with interventional capabilities: which is better for a patient with suspected stroke? JAMA Neurol 74 (5) (2017) 504-506, https://doi.org/10.1001/jamaneurol.201 7.0006.

5 N.J. Kassebaum, M. Arora, R.M. Barber, et al., Global, regional, and national disability-adjusted life-years (DALYs) for 315 diseases and injuries and healthy life expectancy (HALE), 1990-2015: a systematic analysis for the Global Burden of Disease Study 2015, Lancet 388 (2016) 1603-1658, https://doi.org/10.1016/ S01406736(16)31460-X.

6 Sacco RL, Kasner SE, Broderick JP, Caplan LR, Connors JJ, Culebras A, et al. American Heart Association Stroke Council, Council on Cardiovascular Surgery and Anesthesia; Council on Cardiovascular Radiology and Intervention; Council on Cardiovascular and Stroke Nursing; Council on Epidemiology and Prevention; Council on Peripheral Vascular Disease; Council on Nutrition, Physical Activity, and Metabolism. An updated definition of stroke for the 21st century: a statement for healthcare professionals from the American Heart Association/American Stroke Association. Stroke. 2013; 44:20642089.

7 Larrue V, von Kummer RR, Muller A, Bluhmki E. Risk factors for severe hemorrhagic transformation in ischemic stroke patients treated with recombinant tissue plasminogen activator: a secondary analysis of the European-Australasian Acute Stroke Study (ECASS II). Stroke 2001; 32: 438-41.

8 Adams HP Jr, Bendixen BH, Kappelle LJ, Biller J, Love BB, Gordon DL, et al. Classification of subtype of acute ischemic stroke: definitions for use in a multicenter clinical trial. TOAST. Trial of Org 10172 in Acute Stroke Treatment. Stroke. 1993; 24:35-41.

9 The NINDS t-PA Stroke Study Group. Intracerebral hemorrhage after intravenous t-PA therapy for ischemic stroke. Stroke. 1997; 28:2109-2118.

10 Fiorelli M, Bastianello S, von Kummer R, del Zoppo G, Larrue V, Lesaffre E, Ringleb A, Lorenzano S, Manelfe C, Bozzao L, for the ECASS I study group. Hemorrhagic transformation within 36 hours of a cerebral infarct: relationships with early clinical deterioration and 3month outcome in the European Cooperative Acute Stroke Study I (ECASS I) cohort. Stroke. 1999; 30:2280-2284. 